INPLASY

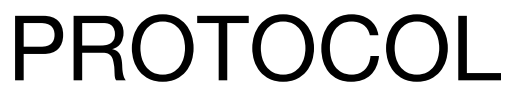

To cite: Xu et al. Comparison of robotic-assisted versus conventional unicompartmental knee arthroplasty for the treatment of single compartment knee osteoarthritis: a meta-analysis. Inplasy protocol 202040014. doi:

10.37766/inplasy2020.4.0014

Received: 03 April 2020

Published: 03 April 2020

Corresponding author: Jiale Zhang

xuketeng1994@163.com

Author Affiliation:

Department of Orthopedics, Clinical Medical Colleg

\section{Support: NSFC}

Review Stage at time of this submission: Risk of bias assessment.

Conflicts of interest: None.

\section{Comparison of robotic-assisted versus} conventional unicompartmental knee arthroplasty for the treatment of single compartment knee osteoarthritis: a meta-analysis

$\mathrm{Xu}, \mathrm{H}^{1}$; Zhang, J2.

Condition being studied: Knee osteoarthritis(OA) is a common disorder characterized by limiting patient physical activities and entertainments[1], the common found in middle-aged and older adults, with more than half of all people over the age of 65 suffering from osteoarthritis[2]. Some researchers have estimated that osteoarthritis will be the fourth most disabling disease by 2020[3]. UKA can be applied in the treatment of single compartment knee osteoarthritis (OA) after the failure of conservative therapy[4]. UKA as a treatment for isolated compartment osteoarthritis (OA) has obvious benefits over total knee arthroplasty(TKA), such as less blood loss in surgery, preserving the unaffected side, faster recovery time, improved functional outcome, perioperative morbidity, and more normal gait[5-7]. These advantages are offset by the higher rate of complications(osteoarthritis of the contralateral compartment implant loosening and revision) UKA compared to that of TKA [8, 9]. These complications commonly result from component malposition and limb malalignment[6, 10, 11]. So, roboticassisted orthopedic surgery was implemented to improve preoperative planning, the accuracy of implant positioning, and precision in bone cuts during UKA. Survival of UKA is mainly dependent on component position; malalignment of the prosthesis may result in poor post-operative function and progressive wear leading to early revision surgery.

INPLASY registration number: This protocol was registered with the International Platform of Registered Systematic Review and Meta-Analysis Protocols (INPLASY) on 03 April 2020 and was last updated on 03 April 2020 (registration number INPLASY202040014.

\section{INTRODUCTION}

Review question / Objectives: Whether more satisfying clinical and radiographic results could be obtained in the acquired robotic system when compared with the conventional method.. 
Condition being studied: Knee osteoarthritis(OA) is a common disorder characterized by limiting patient physical activities and entertainments[1], the common found in middle-aged and older adults, with more than half of all people over the age of 65 suffering from osteoarthritis[2]. Some researchers have estimated that osteoarthritis will be the fourth most disabling disease by 2020[3]. UKA can be applied in the treatment of single compartment knee osteoarthritis (OA) after the failure of conservative therapy[4]. UKA as a treatment for isolated compartment osteoarthritis (OA) has obvious benefits over total knee arthroplasty(TKA), such as less blood loss in surgery, preserving the unaffected side, faster recovery time, improved functional outcome, perioperative morbidity, and more normal gait[5-7]. These advantages are offset by the higher rate of complications(osteoarthritis of the contralateral compartment implant loosening and revision) UKA compared to that of TKA [8, 9]. These complications commonly result from component malposition and limb malalignment $[6,10$, 11]. So, robotic-assisted orthopedic surgery was implemented to improve preoperative planning, the accuracy of implant positioning, and precision in bone cuts during UKA. Survival of UKA is mainly dependent on component position; malalignment of the prosthesis may result in poor post-operative function and progressive wear leading to early revision surgery.

\section{METHODS}

Participant or population: Patients have undergone robotic-assisted or conventional Unicompartmental Knee Arthroplasty.

Intervention: Patients have undergone robotic-assisted Unicompartmental Knee Arthroplasty.

Comparator: Patients have undergone conventional Unicompartmental Knee Arthroplasty.
Study designs to be included: Controlled Trials.

Eligibility criteria: (1) Studies compared the robotic-assisted UKA and conventional UKA. (2) Clinical or radiographic outcomes were not limited to pool. (3) Studies published in English.

Information sources: Embase, Medline, Web of Science, Cochrane databases were searched to retrieve related studies updated in January 2020.

Main outcome(s): limb alignment of satisfactory ranges.

Additional outcomes: AKSS (American knee society knee score), Range of Motion(ROM), Surgical Time (minutes); Tibial axial; limb alignment of satisfactory ranges, pain, revision rate, total complications.

Quality assessment / Risk of bias analysis: The quality of the non-RCTs studies was assessed according to the Downs and Black [20] and the Newcastle-Ottawa Scale (NOS) quality assessment method. A total NOS score was $9^{*}$ and if the NOS score was over $6^{*}$, it would be considered as higher quality research. A higher score was recognized as better quality research. The 12-item scale was used to assess the quality of the 5 RCTs16. Each item was scored "Yes", "Unclear", or "No". If a trial with a score of more than 7 "Yes" was considered high quality, more than 4 but no more than 7 was considered moderate quality, and no more than 4 was considered low quality. Any different opinions were resolved by a third reviewer.

Strategy of data synthesis: Statistical heterogeneity of data was evaluated by using Cochran's $\mathbf{Q}$ statistic. If statistical $\mathbf{Q}$ statistic $(P<0.10)$ was considered to be significant heterogeneous among studies, a random-effects model was performed, if not, a fixed-effects model was used. If the heterogeneity of a parameter was over $85 \%$, the meta-analysis was not performed. The results of continuous data were applied to the mean difference with $95 \%$ 
confidence interval (CI). For dichotomous data, the Odd ratio (OR) was calculated using the Mantel-Haenszal method, mean difference and standardized mean difference were considered statistically significant at the $P<0.05$ level. Data analysis was carried out by using Review Manager 5.3. Sensitivity analysis was performed to assess the results through the exclusion of eligible studies once time.

Subgroup analysis: Owing to the limited information, subgroup analyses were just conducted on follow-up ( $<2$ years, $\geq 2$ years).

Sensibility analysis: One study was individual deleted each time to observe its influence on the pooled MD or OR. The results showed that no study could substantially affect the pooled MD or OR in the present meta-analysis.

Coutries involved: China.

Keywords: robotic-assisted; Knee Osteoarthritis; Unicompartmental arthroplasty; Meta-analysis. 